\title{
Influence Factors on Microleakage of Dental Composite Resin
}

\author{
Shuang Wang ${ }^{\mathrm{a}}$, Zhenzhen $\mathrm{Xu}{ }^{\mathrm{b}}$, Hongna Gaoc, Niannian Tan ${ }^{\mathrm{d}}$, Fujie Zhai ${ }^{\mathrm{e}}$ \\ Binzhou Polytechnic, Shandong Binzhou, 256603, China

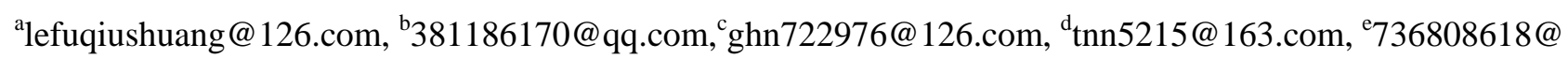 \\ qq.com
}

Keywords: Composite Resin; Microleakage; Influence Factors

\begin{abstract}
Dental composite resin is a kind of material which has been widely used in dental restoration. It was found that the marginal adaptation of the composite resin was slightly misfit and microleakage was easily formed. The harms of microleakage of composite resin was discussed in this paper. The effects of resin matrix and inorganic filler, initiating system, illumination, repair technique and surface treatment on the polymerization shrinkage of composite resin were also analyzed. The paper also discusses the effective measures to reduce micro-leakage of resin, and offers theoretical basis for the clinical application and development of composite resin.
\end{abstract}

\section{Introduction}

Composite resin used for tooth restoration has become the main material due to its good aesthetic and mechanical properties.However, there is a general shrinkage of polymer volume in composite resin and the thermal expansion coefficient of composite resin material is not consistent with that of dental hard tissue. Polymerization shrinkage stress and alternating cold and hot stress lead to edge cracks between tooth and resin material.Invisible substances such as bacteria and food residues cross the interface between the tooth and the prosthesis or filling material, which is clinically called marginal microleakage ${ }^{[1]}$. Microleakage will greatly reduce the success rate of dental restoration, resulting in a variety of adverse effects, such as staining of backfill edge, secondary caries, postoperative sensitivity and even backfill detachment. Microleakage at the edge of restoration is one of the main factors affecting the quality of restoration, and the generation of microleakage is affected by many factors. This paper reviews the harm and influencing factors of microleakage of dental composite resin.

\section{Description of composite resin}

Composite resin is a kind of resin matrix composite material, which is mainly composed of polymerizable resin matrix, inorganic particle filler with reinforcing effect and initiating system. Despite the continuous efforts of researchers and the continuous optimization of composite resin properties, there are still problems such as polymerization shrinkage and edge microleakage ${ }^{[2]}$. The polymerization shrinkage of composite resin is mainly due to the formation of a large number of chemical bonds in the resin matrix during the curing process. The distance between molecules changes from loose to close, resulting in the macroscopic volume shrinkage. Because of the adhesion between the cavity wall and the composite resin material, the shrinkage stress is caused by limiting the volume shrinkage of a material. The thermal expansion coefficient of the composite resin $\left[(14-50) \times 10^{-6} \cdot \mathrm{K}^{-1}\right]$ was significantly higher than that of the tooth hard tissue. In the environment of variable oral temperature, the expansion and contraction of materials and dental tissues are inconsistent, and the resin filling body will suffer fatigue damage under the action of this thermal stress. Polymerization shrinkage stress and thermal stress together form the interface failure stress between the prosthesis and the tooth, which leads to the decrease of the edge fitness between the prosthesis and the tooth. 


\section{Hazards of microleakage}

\subsection{Effect on vital teeth}

After the vital teeth are filled with composite resin, the gap between the filling material and the tooth will cause bacteria and their metabolites to gather here, corrode the material and erode the hard tissue of the tooth at the same time, resulting in secondary caries; pigmentation causes color change at the edge of the pit; adverse stimulation was introduced into the pulp through the dentine tubules, which caused postoperative sensitivity of teeth; the presence of secondary caries may result in loosening and shedding of resin filling materials and even pulpitis ${ }^{[3]}$.

\subsection{Effect on root canal}

The marginal closeness of filling material and dental tissue affects the effect of pulp treatment, and the generation of microleakage is an important reason for the failure of root canal treatment. Swanson et al. have shown in vitro studies that bacteria can enter the treated root canal through cracks at the filling edge, leading to re-infection of the root canal system ${ }^{[4]}$. Infection can also infiltrate into the periapical tissue along the filling material of the root tip, leading to the failure of pulp treatment.

\section{Factors influencing microleakage}

The results show that the main reason of microleakage is that the polymerization shrinkage of composite resin during polymerization and the thermal expansion coefficient of composite resin does not match the tooth structure. The factors influencing shrinkage and thermal expansion coefficient of composite resin polymerization mainly include the following aspects: matrix composition, inorganic filler, initiating system, illumination, repair technology, surface treatment, etc.

\subsection{Resin matrix}

Bisphenol A- dimethacrylate glycidyl ester (Bis-GMA) is the main monomer of traditional composite resin, free radicals initiate polymerization of monomers. The chain growth process changes from the initial linear monomer sliding polymerization to reticular monomer crosslinking. The molecular spacing shrinks and the volume shrinks, resulting in microleakage. In recent years, epoxy resin as the matrix resin has been applied. Epoxy groups are open-loop polymerization ${ }^{[5]}$, and the distance between molecules decreases first and then increases, partially offsetting the volume contraction caused by bonding. Studies have shown that the higher the conversion rate of double bond in the composite resin, the greater the polymerization shrinkage. Therefore, appropriate reduction of the percentage of resin matrix in the material can reduce the double bond conversion rate, thereby reducing the volume shrinkage rate. Compared with Bis-GMA, the new modified methacrylate matrix system ${ }^{[6]}$ (Bis-EMA) reduces two hydrophilic hydroxyl functional groups and significantly reduces the viscosity of the resin, so as to add more fillers and reduce the polymerization shrinkage rate.

\subsection{Inorganic filler}

Adding high strength inorganic filler to the composite resin can significantly improve the mechanical properties of the material, which is the key component of the composite resin in clinical application. It has been proved that the higher the content of inorganic filler and its volume in the resin, the greater the compressive strength and elastic modulus of the material ${ }^{[7]}$. In the process of polymerization, the degree of reduction of molecular spacing is reduced, and the stress during polymerization shrinkage is smaller. At the same time, the thermal expansion coefficient of the composite resin is closer to the tooth, which greatly reduces the adverse effect of thermal stress and significantly improves the edge tightness of the backfill. Adding the right amount of thinner in the resin matrix can increase the content of inorganic filler in the material, however, the content of filler 
should not be too much in order not to affect the performance of the composite resin.

The particle size of inorganic filler affects the physical and mechanical properties and polymerization shrinkage of composite resin. A large number of studies have confirmed that the finer the particle size of the filler, the stronger the physical and mechanical properties of the resin, and the material is not easy to crack in the process of stress. The incidences of secondary caries and the tinting degree of the edge of prosthesis were reduced. Under the same content of inorganic filler, the smaller the particle size, the greater the fluidity ${ }^{[8]}$. The resin with good fluidity is relatively dense after contact with the hard tissue of tooth, which enhances the edge sealing property of the filling resin material and thus reduces the occurrence of microleakage. The polymerization shrinkage of the composite resin can be made up by the water absorption expansion of the material to a certain extent ${ }^{[9]}$. The water absorption of the filler with small particle size is greater than that of the filler with large particle size, which reduces the edge crack and microleakage. Some scholars believe that nano-filler composite resin is superior to other types of resin in reducing microleakage ${ }^{[10]}$. Nano fillers generally consists of nanoparticles and nano particle clusters, the size of the two particles is different, by optimizing the ratio, it can effectively reduce the gap between the filler, increase the packing density, thus increasing the content of filler, and the nanometer composite resin filler can also produce stress relieving point, thus reduce the polymerization shrinkage stress.

\subsection{Initiating system}

The REDOX induced chemical curing composite resin has a long curing time, and the initial material has a large fluidity. The shrinkage of polymerization volume can be compensated by the flow deformation of the filling material. The most commonly used initiator of visible light curing composite resin is camphor quinone, which is sensitive to light with a wavelength of $440 \sim 500 \mathrm{~nm}$. The generation of free radicals increases the polymerization rate of the resin, increases the shrinkage stress, and finally increases the incidence of microleakage.

\subsection{Illumination}

It has been reported in the literature that the illumination mode of the photocured composite resin affects the edge sealing performance of the composite resin material. The main reason of microleakage between the filling material and tooth tissue caused by light is that the resin matrix produces micropores due to irradiation.The ionic bond between the inorganic fillers was destroyed, and the bridging relationship between the fillers collapsed and split, resulting in the contraction of energy dispersion. The polymerization shrinkage of photocured composite resin has the direction of phototaxis shrinkage and centrality shrinkage. Some scholars have pointed out that the decrease of the initial polymerization rate of resin will make the filling material have long-term fluidity at the initial stage of curing, and the larger flow deformation can compensate for the volume shrinkage of resin polymerization. Therefore, compared with the traditional illumination technology of constant intensity and irradiation time, low-light guided illumination technology and indirect illumination technology can slow down the initial speed of resin curing, improve the compensation effect, reduce polymerization shrinkage and improve the edge tightness. The research shows that the irradiation Angle perpendicular to the filling material can greatly reduce the occurrence of microleakage.

\subsection{Repair technique}

According to the clinical repair process, the composite resin can be divided into direct filling composite resin and indirect repair composite resin. Horizontal stratification curing is a common method of direct filling stratification curing, but studies have shown that stratification oblique curing can effectively improve the edge microleakage due to the inconsistent shrinkage direction of each layer of curing. At present, the indirect repair technology of composite resin, such as imbedding technology, has developed quite mature.The curing process of resin is carried out in an external photocuring box or a hot press polymerizer, which can be cured in multiple directions for a long time. This technology can not only improve the mechanical properties of the material, but also improve the degree of polymerization. The filling material adheres to the cavity by adhesive, which 
can effectively reduce the micro leakage at the edge.

Preheating composite resin filling technology has been proved to have good edge suitability ${ }^{[11]}$. Resin after repeated cyclic heating and cooling (room temperature to $60{ }^{\circ} \mathrm{C}$ ) and extend the preheating, allow it to increase liquidity, significantly reduced the micro leakage after filling. Wagner et al. also found that the preheating of the resin would increase the speed of the filling material to return to the original shape, so the preheated resin should be illuminated in time after filling to prevent the resin from returning to deformation and causing edge micro-cracks.

\subsection{Surface treatment}

The stronger the adhesion between the composite resin and the cavity, the stronger the resistance to the stress caused by the polymerization shrinkage of the resin, and the smaller the possibility of microleakage. The bonding effect is affected by acid etching technology ${ }^{[12]}$. Acid etching works by demineralizing enamel and dentin surfaces to form micropores, which increase the area of contact between the adhesive and tooth tissue. At the same time, the adhesive penetrates into the micropores to form a large number of resin protrusions, which enhances the mechanical Mosaic effect and greatly increases the adhesive force. The acid etching agent etches the tooth enamel better, so the acid etching and washing adhesive has a higher bonding strength of tooth enamel than the self-etching adhesive. Additional acid etching of enamel, especially selective enamel acid etching combined with self-etching can effectively improve adhesion when filling deep dentin cavities $^{[13]}$.

\subsection{Surface sealant}

After filling and curing the composite resin, the sealant is applied on the surface of the tooth-filling body to effectively seal the cracks between the resin filling material and the tooth hard tissue $^{[14]}$. The surface sealant has good fluidity, strong permeability, and forms close chimeric with resin and tooth tissue. When the temperature changes, the volume changes little and it is not easy to fall off. Surface sealant not only has good wettability to dentin and reduce the damage of grinding and polishing to tooth tissue, but also reduce the voids on the surface of resin material, make the surface of resin and tooth smooth, reduce the deposition of bacteria and metabolites, and reduce the occurrence of microleakage.

\section{Expectation}

Because of its convenient operation and beautiful appearance, composite resin for dental repair has an irreplaceable role in dental repair, and is welcomed by dental professionals and patients. How to improve the physical and chemical properties, mechanical and biological properties of materials has been the focus of scholars in recent years. Edge compactness is one of the important indexes to evaluate the properties of filling materials. It is an important aspect of future research to improve and replace the materials in view of the influencing factors of polymerization shrinkage of composite resin. For example, improve the matrix composition, and vigorously develop new multi-functional high-reactive monomers and resin systems; the nanometer inorganic filler system with good biocompatibility and bioactivity was developed; to design and prepare novel initiator with high sensitivity and non-toxicity, and to reduce the initial polymerization rate of resin appropriately; adopt the new curing method, ensure the material and curing device match, improve the resin curing early flow deformation; the second stage of curing resin treatment makes the polymerization more complete; mature and effective acid etching agent technology and good adhesives and surface sealants were used. High quality and biocompatible resin restorations can be obtained only by strictly controlling various influencing factors. It is believed that the composite resin will obtain satisfactory clinical effect and be widely used in clinical practice for the benefit of mankind. 


\section{Acknowledgements}

Fund Program: Binzhou Vocational College Science and Technology Development Project (Project No. 2018YJKT01)

Project name: The cytotoxicity of tooth restorative nano-hydroxyapatite composite resin in vitro was evaluated by dentin barrier method

\section{References}

[1] Sharafeddin F, Feizi N. Evaluation of the effect of adding micro-hydroxyapatite and Irnano-hydroxyapatite on the microleakage of conventional \rand resin-modified Glass-ionomer CI V restorations [J]. Journal of Clinical and Experimental Dentistry, 2017:0-0.

[2] Tavangar M, Davalloo R T, Darabi F, et al. A Comparative Evaluation of Microleakage of Two Low-Shrinkage Composites with a Conventional Resin Composite: an In Vitro Assessment [J]. Journal of Dentistry, 2016, 17(1):55-61.

[3] Sharma R D, Sharma J, Rani A. Comparative evaluation of marginal adaptation between nanocomposites and microhybrid composites exposed to two light cure units.[J]. Indian Journal of Dental Research Official Publication of Indian Society for Dental Research, 2011, 22(3):495.

[4] Vijay R, Indira R. Effect of glass-ionomer cement as an intra-canal barrier in post space prepared teeth: An in vitro study [J]. Journal of Conservative Dentistry, 2009, 12(2):65-68.

[5] Jiang T, Shuang L I, Zhao H, et al. Research progress of epoxy resin modified by chemical copolymerization [J]. Journal of Hubei University, 2017.

[6] Silva E M, Miragaya L, Noronha-Filho J D, et al. Characterization of an experimental resin composite organic matrix based on a tri-functional methacrylate monomer [J]. Dental Materials Journal, 2016, 35(2):159.

[7] Fronza B , Ayres A , Pacheco R , et al. Characterization of Inorganic Filler Content, Mechanical Properties, and Light Transmission of Bulk-fill Resin Composites[J]. Operative Dentistry, 2017:16-024-L.

[8] Fenglei Z, Liang C, Congyun H. Research Progress of Inorganic Fillers in Dental Composite Resin [J]. Materials Review, 2016.

[9] Versluis A, Tantbirojn D, Lee M S, et al. Can hygroscopic expansion compensate polymerization shrinkage? Part I. Deformation of restored teeth [J]. Dental Materials Official Publication of the Academy of Dental Materials, 2011, 27(2):126-133.

[10] Teymoornezhad K , Alaghehmand H , Daryakenari G , et al. Evaluating the Microshear Bond Strength and Microleakage of Flowable Composites Containing Zinc Oxide Nano-particles[J]. Electronic Physician, 2016, 8(11):3289-3295.

[11] Demirbuga S, Ucar F I, Cayabatmaz M, et al. Microshear bond strength of preheated siloraneand methacrylate-based composite resins to dentin [J]. Scanning, 2015, 38(1):63-69.

[12] Tsujimoto A, Barkmeier W W , Takamizawa T, et al. The Effect of Phosphoric Acid Pre-etching Times on Bonding Performance and Surface Free Energy with Single-step Self-etch Adhesives [J]. Operative Dentistry, 2016, 41(4):441.

[13] Chunxia Y, Bingxin H, Lijing L, et al. In vitro experimental study of selective enamel etching combined with self-etching adhesive resin edge microleakage $[\mathrm{J}]$. Laboratory medicine and clinical, 2016, 13(16).

[14] Hepdeniz O K , Temel U B , Ugurlu M , et al. The effect of surface sealants with different filler content on microleakage of Class V resin composite restorations [J]. European Journal of Dentistry, 2016, 10(2):163-169. 